\title{
Matt Tomlinson, Debra McDougall (eds.), Christian Politics in Oceania
}

New York/Oxford, Berghahn Books, coll. « ASAO Studies in Pacific Anthropology ", vol. 2, 2013, 260 p.

Illustrations, cartes, bibliographie, index.

\section{Benoît Vermander}

\section{(2) OpenEdition}

12 Journals

\section{Édition électronique}

URL : http://journals.openedition.org/assr/26578

DOI : $10.4000 /$ assr.26578

ISSN : $1777-5825$

Éditeur

Éditions de l'EHESS

\section{Édition imprimée}

Date de publication : 31 décembre 2014

Pagination : 302

ISBN : 978-2-7132-2467-6

ISSN : 0335-5985

Référence électronique

Benoît Vermander, « Matt Tomlinson, Debra McDougall (eds.), Christian Politics in Oceania », Archives de sciences sociales des religions [En ligne], 168|2014, mis en ligne le 21 mai 2015, consulté le 22 septembre 2020. URL : http://journals.openedition.org/assr/26578; DOI : https://doi.org/10.4000/ assr.26578

Ce document a été généré automatiquement le 22 septembre 2020.

(c) Archives de sciences sociales des religions 


\section{Matt Tomlinson, Debra McDougall (eds.), Christian Politics in Oceania}

New York/Oxford, Berghahn Books, coll. « ASAO Studies in Pacific Anthropology ", vol. 2, 2013, 260 p.

Illustrations, cartes, bibliographie, index.

\section{Benoît Vermander}

\section{RÉFÉRENCE}

Matt Tomlinson, Debra McDougall (eds.), Christian Politics in Oceania, New York/ Oxford, Berghahn Books, coll. «ASAO Studies in Pacific Anthropology », vol. 2, 2013, $260 \mathrm{p}$. 
1 Il faut a border le présent volume en ayant à l'esprit les perspectives ouvertes par des travaux comme ceux de Fenella Cannell (The Anthropology of Christianity, 2006, volume collectif): ces dernières années, la question de la "différence " induite par la christianisation d'une société a captivé nombre d'anthropologues, en même temps qu'ils en précisaient et diversifiaient les termes. Elle est d'une importance particulière pour les sociétés océaniennes, lesquelles sont, statistiquement, les plus «chrétiennes» du globe, et connaissent un degré d'intervention des Églises dans la vie publique dont on connaît peu d'exemples ailleurs. Identités chrétienne et nationale sont souvent affirmées d'un seul tenant dans la constitution de ces

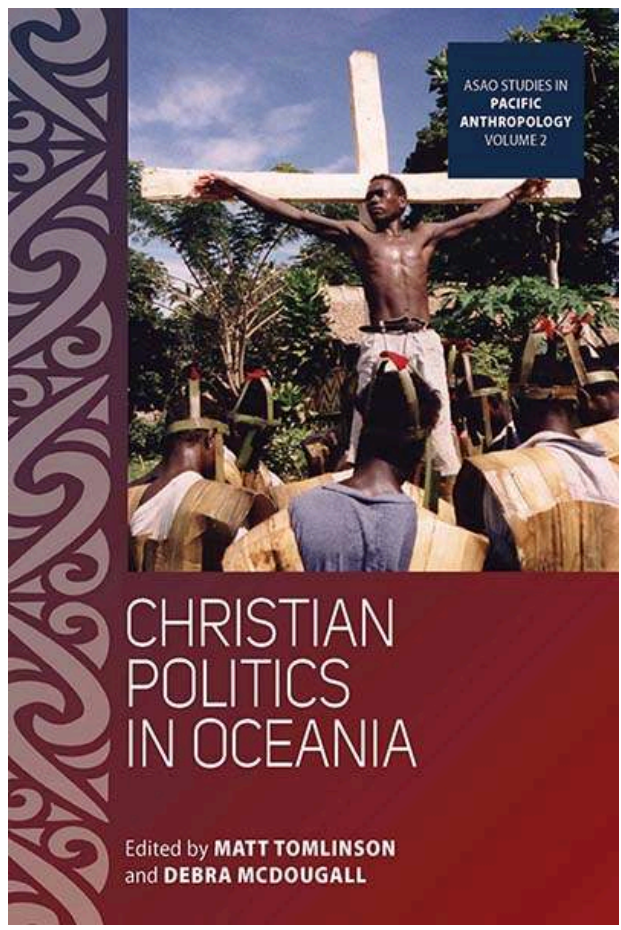
nouveaux États (il en est ainsi, à des degrés divers, pour Samoa, Nauru, Kiribati, les îles Marshall, la Papouasie-Nouvelle-Guinée, les îles Salomon, Tuvalu et Tonga). Les contributions ici rassemblées explorent donc les liens à la fois intenses et complexes noués entre le « politique » et le « religieux » dans un ensemble océanien qui fut aussi marqué ces vingt ou trente dernières années par l'instabilité politique et sociale. Les perspectives adoptées sont résolument anthropologiques, privilégiant l'étude de cas et la multiplicité des niveaux d'analyse. De ce fait même, les situations couvertes sont loin de correspondre à toutes celles observables dans cet ensemble régional, et l'étude des mouvements situés « à la marge » est plus nourrie que celle des organisations de facture plus traditionnelle. Il faut du reste lire l'introduction à ce volume en parallèle avec une contribution située plus avant : John Barker se livre à une revue critique de la littérature consacrée à la "politique chrétienne » dans le contexte de la Papouasie-Nouvelle-Guinée pour appeler en finale à ouvrir davantage le champ aux approches de la sociologie ou de la science politique plutôt qu'à vouloir maintenir son « formatage » initial par l'anthropologie, la discipline qui domine encore le paysage régional des sciences sociales. Ce n'est pas l'un des moindres intérêts de l'ouvrage que d'être ainsi à la fois solidement inscrit dans une tradition et d'en questionner avec grande liberté de ton les méthodes, les acquis et les frontières.

Dans un premier chapitre, Courtney Handman s'intéresse à trois églises protestantes en compétition sur le territoire d'une vallée de Papouasie-Nouvelle-Guinée, dans la province de Morobe. De l'Église luthérienne sortent progressivement la New Life Church, fruit d'un renouveau charismatique de la fin des années 1970, puis la Reformed Gospel Church, créée en réaction au localisme de la précédente. Handman analyse leurs interactions et les enjeux sous-jacents au travers, d'une part d'une dispute légale autour d'un terrain comprenant une piste d'atterrissage, d'autre part de l'usage des instruments (tambours et guitares notamment) dans la pratique liturgique. Le conflit foncier est manifestement multidimensionnel et il est ici résumé de façon quelque peu 
elliptique. L'auteur note que les Églises n'apparaissent pas directement dans les disputes juridiques, utilisant plutôt à leur profit les droits coutumiers résultant de généalogies concurrentes, ou les détournant par intervention venue d'en haut - tout en se défendant d'intervenir dans le "politique ». En même temps, les projets fonciers en compétition sont porteurs de visions religieuses distinctes, voire opposées, l'une aspirant à bâtir la communauté locale en "ville sur la colline » tandis que l'autre vise à l'ouvrir sur les réseaux chrétiens de l'ensemble du pays. L'analyse des pratiques instrumentales liturgiques est plus développée, et est riche d'implications: le refus initial de l'Église luthérienne d'utiliser les tambours disparaît peu à peu une fois que la New Life Church en a popularisé la pratique, et son introduction accompagne l'évolution du répertoire qui résulte de la traduction du Nouveau Testament en langue locale. Mais dans l'Église luthérienne seule les femmes jouent du tambour durant les services, ce en conformité avec la tradition locale qui voit dans le christianisme une fraicheur ou douceur de mœurs («cold» et «cool») opposée à la « chaleur » des mœurs anciennes. Et l'usage du tambour est contrebalancé par celui de fanfares et autres dispositions instrumentales. Le style mâle et bruyant de la New Life Church (où le tambour, joué par les hommes, prédomine) finit par susciter en réaction son exact opposé dans celui de la Reformed Gospel Church, centré sur des textes chantés successivement en plusieurs langues, accompagnés exclusivement par des guitares et tambourins. La RGC confère aussi un rôle essentiel aux chœurs de petites filles, la frange la plus marginale de la communauté sociale, poussant ainsi l'exaltation de la " coolness » à un point qui semble parfois menacer son enracinement social. L'auteur tire profit de son analyse pour questionner l'analyse classique de Niebuhr (The Social Sources of Denominationalism, 1929) décrivant l'incessant passage de "l'Église » à la « secte », de l'institution politique à la radicalité de choix individuels librement associés. Elle montre que l'imbrication du « politique » et du « religieux » dans l'espace-temps qu'elle analyse correspond moins à des phases bien définies qu'à des perceptions différentes des interactions en cours, telles que les acteurs eux-mêmes les éprouvent et les expriment. Si la conclusion est trop ramassée pour être entièrement claire, la subtilité de l'analyse des formes sociales et liturgiques développée au cours de l'article est remarquable.

3 La contribution de Michael Scott a pour cadre l'île de Makira, au sud-est de Guadalcanal, dans les îles Salomon. Elle examine la rumeur qui situe à la pointe nordouest de l'île le siège d'une armée souterraine (les figures narratives qui la construisent empruntent à l'imagerie des bases américaines, aux figures mythologiques traditionnelles et aux mémoires des conflits ayant déchiré les îles Salomon entre 1998 et 2003). Ces rumeurs contribuent à construire l'île en une entité politique spécifique, indépendante des lignées matrilinéaires, tout en "révélant » en Makita le véritable théâtre d'Armageddon. Les identités religieuses comme les perceptions politiques concourent à faire de l'armée souterraine celle qui établira « les cieux sur la terre » ou bien (en exact opposé) «la base de Satan dans le Pacifique», pire même : une "autre Rome ». L'analyse de Scott, fortement théorisée, emprunte aux travaux du cercle de Bakhtin pour faire des discours développés autour de cette armée "un monde dialogiquement construit » (p. 50). L'analyse de l'auteur souffre quelque peu de citer essentiellement les interviews d'un seul couple d'adventistes du Septième Jour par lui conduites - mais il est vrai que l'expertise qu'il a développée de longue date sur Makita l'autorise à nous proposer en cette contribution une sorte d'arrêt sur images, ajoutant ainsi sa propre strate aux constructions dialogiques qu'il pourchasse et décrypte avec 
ténacité. L'analyse de discours ici poursuivie a notamment le mérite de pointer l'ambiguïé de l'opposition apparemment irréductible entre représentations " céleste " et "satanique", les arcanes du mythe révélant les ambivalences habitant ses producteurs.

Matt Tomlinson retrace avec clarté les positions des Églises de Fidji devant les coups d'État qui ont scandé la vie politique depuis 1987, les trois premiers appuyés par l'Église Méthodiste qui regroupe la très grande majorité des Fidjiens « de souche ». La situation s'inverse avec le coup d'État de 2006, auquel s'opposent farouchement les Méthodistes, alors qu'il est soutenu plus ou moins ouvertement par l'évêque catholique mais aussi par les "Nouveaux Méthodistes", un mouvement dont la rhétorique fait l'objet du cœur de la contribution. Tomlinson montre l'hésitation affectant l'exaltation conjointe de la «nouveauté " chrétienne et de la «tradition» ethnique dans le vocabulaire méthodiste, et pointe en contraste le choix résolu de la «nouveauté » effectué par les fondateurs de la nouvelle Église, un choix qui sert à justifier le coup d'État et le soutien à un gouvernement qui intitule son journal «Nouvelle Aurore». En juin 2009, la «croisade chrétienne» des forces de police, lesquelles sont dirigées par l'un des fondateurs des Nouveaux Méthodistes, donne corps au discours politico-religieux qui s'affirme. Mais l'imbrication trop explicite des objectifs provoque en finale la dislocation de la nouvelle Église, sur l'instigation d'un gouvernement qui doit asseoir autrement sa légitimité.

5 L'étude d'Annelin Eriksen sur Églises et politique à Vanuatu procède différemment des précédentes. Elle débute par un résumé incisif des approches théoriques qui questionnent la distinction entre État et société civile, puis les applique à son champ de travail pour offrir une approche alternative à celle qui verrait dans les Églises l'une des expressions de la société civile « en face » de l'État, quand même celui-ci serait décrit comme "faible» ou "manqué ». L'auteur montre d'abord que, durant la période coloniale, organes d'État, économie de plantation et institutions chrétiennes travaillaient de façon tellement imbriquée au respect et à l'intériorisation de la moralité publique que les institutions ecclésiales étaient celles-là mêmes qui instillaient un sens fort de ce qu'est l'État et de sa mission. L'indépendance a coïncidé avec la fin de l'économie de plantation et la multiplication des acteurs (nationaux et internationaux) capables de produire des "effets étatiques ». À l'intérieur d'un "État flottant» (freefloating state) des structures ecclésiales ont désormais charge d'assurer des « missions de gouvernance » dont on ne saurait rendre compte en les décrivant comme le simple exercice du pouvoir d'initiative de la société civile mais dont il faut savoir reconnaître qu'elles s'intègrent de plain-pied dans ce qu'on a coutume d'appeler "pouvoir étatique ». Deux cas illustrent brièvement cette approche, laquelle entend mettre en lumière la souplesse et l'importance des missions assumées par les Églises, anciennes ou nouvelles, dans la structure générale de "l'État flottant». La clarté de la contribution fait d'elle une excellente ressource pour tout travail comparatiste sur ce thème.

6 Debra McDougall retrace le parcours de deux jeunes hommes originaires des îles Salomon, l'un ayant "navigué » entre trois Églises évangéliques, l'autre étant passé d'une autre Église évangélique à l'islam sunnite, tout en ayant conservé le vocabulaire et les références de sa première appartenance. Elle illustre ainsi la fluidité sociale induite par la quête religieuse, dans un archipel qu'on décrit souvent comme étroitement régi par les liens croisés tissés ces cent dernières années entre 
appartenances religieuse et ethnique. La contribution de Geoffrey White a également pour cadre les îles Salomon : l'île de Santa Isabel, dont la très grande majorité de la population appartient à l'Église anglicane de Mélanésie, est le lieu d'un projet de revitalisation du rôle des chefs traditionnels dirigé par le Programme des Nations Unies pour le développement (projet auquel l'auteur de l'article a participé). White décrit en parallèle l'élévation d'un évêque à la dignité de chef suprême et la consécration de son successeur, arborant les insignes de chef coutumier avant d'en être dévêtu durant le rituel d'ordination. L'auteur analyse avec subtilité les différents registres rituels et politiques utilisés pour refonder et harmoniser les niveaux d'autorité induits par la coutume, la politique étatique et l'identité religieuse.

7 Joel Robbins revient sur son travail de terrain parmi les Urapnin (un groupe linguistique de Papouasie-Nouvelle-Guinée d'environ 390 personnes), s'interrogeant sur la raison pour laquelle ce groupe entièrement converti à une forme d'évangélisme à forte tendance millénariste garde néanmoins une séparation stricte entre " politique " et « religion» quant aux autorités en charge et aux processus de négociation. Robbins voit les Urapnin comme « une secte devenue aussi grande qu'une société» (p. 205). De ce fait même, la gestion des affaires sociales sur le modèle de la secte religieuse rendrait impossible la poursuite de la vie normale. La séparation des pouvoirs est accompagnée de divisions temporelles : aux périodes d'exaltation millénariste succède immanquablement le retour au cours ordinaire des choses.

8 La postface de Webb Keane clôt excellemment ce volume. L'auteur relève l'importance des considérations proprement théologiques dans les jugements et les décisions effectués par les acteurs qui font l'objet des diverses contributions, en même temps qu'il signale l'influence sociale et l'originalité des « théologies politiques » océaniennes. Il insiste notamment sur la diversité des représentations temporelles et historiques que différentes théologies politiques nourrissent aujourd'hui, très concrètement, en Océanie. Sur le plan spatial, les «communautés imaginaires» formées par l'appartenance ecclésiale peuvent aussi se révéler plus attractives que celles suscitées par des État-nations en gestation difficile. En général, les appartenances religieuses océaniennes valorisent à l'extrême les catégories de choix, d'initiative et d'intervention, coupant en transversale les structures politiques induites par la mise en place d'État-nations "modernes", et favorisant "une totalisation conceptuelle de la vie morale» (p. 221). Si les conclusions énoncées peuvent sembler parfois elliptiques, elles donnent leur cohérence aux aperçus suggérés par les différentes contributions. Enfin, il nous faut souligner la qualité éditoriale de ce volume, laquelle en accroît encore la réussite d'ensemble. 